\title{
The strongest vector space topology is locally convex on separable linear subspaces
}

\author{
by W. ŻELAZKo (Warszawa)
}

Wtodzimierz Mlak in memoriam

\begin{abstract}
Let $X$ be a real or complex vector space equipped with the strongest vector space topology $\tau_{\max }$. Besides the result announced in the title we prove that $X$ is uncountable-dimensional if and only if it is not locally pseudoconvex.
\end{abstract}

Let $X$ be a real or complex vector space. An F-seminorm on $X$ is a function $x \mapsto\|x\|$ satisfying the following conditions:

(i) $\|0\|=0$ and $\|x\| \geq 0$ for all $x$ in $X$.

(ii) $\|t x\|=\|x\|$ for all $x$ in $X$ and all scalars $t$ with $|t|=1$.

(iii) $\|x+y\| \leq\|x\|+\|y\|$ for all $x, y \in X$.

(iv) $\lim _{n} t_{n} x=0$ for all $x$ in $X$ and all seqences $\left(t_{n}\right)$ of scalars tending to zero.

(v) $\lim _{n} t x_{n}=0$ for all scalars $t$ and all sequences $\left(x_{n}\right)$ of elements of $X$ satisfying $\lim _{n} x_{n}=0$.

An $F$-seminorm $\|\cdot\|$ is said to be $p$-homogeneous, $0<p \leq 1$, if the conditions (iv) and (v) are replaced by

(vi) $\|t x\|=|t|^{p}\|x\|$ for all $x$ in $X$ and all scalars $t$.

In case when $p=1$ it is the familiar homogeneity condition. In this case we call it just a seminorm.

It is well known that any vector space topology $\tau$ on $X$ is given by means of a family $\mathcal{F}(\tau)$ of $F$-seminorms (see [3], Theorem 2.9.2). This means that a net $\left(x_{\alpha}\right)$ of elements of $X$ tends to zero in the topology $\tau$ if and only

1991 Mathematics Subject Classification: Primary 46A16.

Key words and phrases: topological vector spaces, locally pseudoconvex spaces, locally convex subspaces.

Supported by the KBN grant No 220079203. 
if $\lim _{\alpha}\left\|x_{\alpha}\right\|=0$ for all $\|\cdot\|$ in $\mathcal{F}(\tau)$. If all $F$-seminorms in $\mathcal{F}(\tau)$ are p-homogeneous (with $p$ depending upon $\|\cdot\|$ ) the space $(X, \tau)$ is said to be locally pseudoconvex. For $\mathcal{F}(\tau)$ we can always take the family of all $F$ seminorms which are continuous in the topology $\tau$. Note that an $F$-seminorm $\|\cdot\|$ is continuous in the topology given by means of some family $\mathcal{F}(\tau)$ if and only if there are a finite number of seminorms $\|\cdot\|_{1}, \ldots,\|\cdot\|_{n}$ in $\mathcal{F}(\tau)$ with the property that for each positive $\varepsilon$ there is a positive $\delta$ such that whenever $\max \left\{\|x\|_{1}, \ldots,\|x\|_{n}\right\}<\delta$ then $\|x\|<\varepsilon$ for all $x$ in $X$.

Each vector space $X$ has the strongest (maximal) vector space topology given by means of all $F$-seminorms. We shall denote it by $\tau_{\max }$. We can also consider the maximal $p$-convex topology $\tau_{\max }^{p}$ (with $p$ satisfying $0<p \leq 1$ ) given by means of all $p$-homogeneous seminorms, and the topology $\tau_{\max }^{q+}$ $(0 \leq q<1)$ given by means of all $p$-homogeneous seminorms for all $p$ satisfying $q<p \leq 1$. Note that all $q$-homogeneous seminorms, $p \leq q \leq 1$, are continuous in the topology $\tau_{\max }^{p}$. This follows from the fact that for any $q$-homogeneous seminorm $\|\cdot\|$ the seminorm $x \mapsto\|x\|^{p / q}$ is $p$-homogeneous whenever $0<p \leq q$.

Let $\tau_{1}$ and $\tau_{2}$ be two vector space topologies on $X$. Writing $\tau_{1} \leq \tau_{2}$ if $\tau_{2}$ is stronger than $\tau_{1}$ (every $\tau_{1}$-continuous $F$-seminorm is $\tau_{2}$-continuous, or every $\tau_{1}$-open set is open in the topology $\tau_{2}$ ) we see that $\tau_{\max }^{\mathrm{LC}} \leq \tau_{\max }^{p+} \leq$ $\tau_{\max }^{p} \leq \tau_{\max }$ for $0<p<1$ and $\tau_{\max }^{p} \leq \tau_{\max }^{q+}$ for $0 \leq q<p \leq 1$. We also see that $\tau_{\text {max }}^{\mathrm{LC}}$ and $\tau_{\max }^{0+}$ are respectively the strongest locally convex and locally pseudoconvex topologies on $X$.

Since for every $x \neq 0$ in $X$ there is a linear functional $f$ with $f(x) \neq 0$ so that the map $x \mapsto|f(x)|$ is a seminorm satisfying $|x| \neq 0$, we see that the topology $\tau_{\max }^{\mathrm{LC}}$ and the stronger topologies $\tau_{\max }^{p}, \tau_{\max }^{q+}, \tau_{\max }$ are Hausdorff. It is known that the topology $\tau_{\max }^{\mathrm{LC}}$ is complete (every Cauchy net is convergent, see [8], Example on p. 56; cf. also [3], Proposition 6.6.7). Also the topologies $\tau_{\max }^{p}$ and $\tau_{\max }^{q+}$ are complete for $0<p \leq 1$ and $0 \leq q<1$ (see [5]). In [5] it is shown that if the dimension (the cardinality of a Hamel basis) of $X$ is uncountable then all topologies $\tau_{\max }^{p}, \tau_{\max }^{q+}$ are different while they coincide whenever the dimension is at most countable. As a consequence, in [5] a complete non-locally convex topological vector space was obtained such that every separable subspace is locally convex. Here we shall offer simplified proofs of these results by showing that the topology $\tau_{\max }$ is also complete and coincides with $\tau_{\max }^{\mathrm{LC}}$ on countable-dimensional spaces. These results, however, are known and follow from Propositions 4.4.3 and 6.6.9 of [3] (see also [11], p. 213). The author is greatly indebted to Hans Jarchow for calling his attention to this fact. The proofs presented here are different and more elementary. We shall also show that all topologies under discussion are different if the dimension of $X$ is uncountable. For basic facts concerning topological vector spaces the reader is referred to [1]-[4] and [6]-[11]. 
We now construct a certain family $\mathcal{F}_{1}$ of $F$-seminorms which give the topology $\tau_{\max }$. Consider the family $\mathcal{S}$ of all continuous non-decreasing functions on the real closed half-line $\mathbb{R}_{+}$of all non-negative real numbers such that

(1) $\quad f(0)=0$ and $f\left(t_{1}+t_{2}\right) \leq f\left(t_{1}\right)+f\left(t_{2}\right)$ for all $t_{1}, t_{2} \geq 0$.

Let $\left(h_{i}\right)_{i \in J}$ be a Hamel basis for $X$, so that every element $x \in X$ can be uniquely written in the form $x=\sum_{i \in J} g_{i}(x) h_{i}$, where only finitely many scalar coefficients $g_{i}(x)$ are different from zero. Clearly the maps $x \mapsto g_{i}(x)$ are linear functionals on $X$. Consider a map $i \mapsto f_{i} \in \mathcal{S}, i \in J$. To each such map there corresponds an $F$-seminorm on $X$ given by the formula

$$
|x|=\sum_{i \in J} f_{i}\left(\left|g_{i}(x)\right|\right)
$$

this is a well defined function on $X$ and an easy proof that it is an $F$ seminorm is left to the reader. Denote by $\mathcal{F}_{1}$ the family of all $F$-seminorms of the form (2).

We now show that each $F$-seminorm on $X$ is continuous with respect to some $F$-seminorm of the form $(2)$, so that $\mathcal{F}_{1}$ gives the topology $\tau_{\max }$. In fact, let $\|\cdot\|$ be an arbitrary seminorm on $X$. Put $\|x\|_{1}=\max _{0 \leq t \leq 1}\|t x\|$. Using the properties (i)-(iv) we easily see that $\|\cdot\|_{1}$ is an $F$-seminorm on $X$; moreover, the map $|t| \mapsto\|t x\|_{1}$ is non-decreasing and $\|x\| \leq\|x\|_{1}$ for all $x$ in $X$ (actually both $F$-seminorms are equivalent, see [8], Theorem 1.2.2). Now by (iii) we obtain

$$
\|x\| \leq\|x\|_{1}=\left\|\sum_{i \in J} g_{i}(x) h_{i}\right\|_{1} \leq \sum_{i \in J}\left\|g_{i}(x) h_{i}\right\|_{1}=|x| .
$$

Thus $|\cdot|$ is of the form $(2)$ with $f_{i}(t)=\left\|t h_{i}\right\|_{1}$ (one easily sees that these functions $f_{i}$ are in $\mathcal{S}$ ). The formula (3) implies that $\|\cdot\|$ is continuous with respect to $|\cdot|$. Since $\|\cdot\|$ was chosen arbitrarily and $|\cdot| \in \mathcal{F}$, the family $\mathcal{F}_{1}$ gives the topology $\tau_{\max }$.

Let $\left(h_{i}\right)_{i \in J}$ be a fixed Hamel basis in $X$. Define the support of $x \in X$ by

$$
\operatorname{supp}(x)=\left\{i \in J: g_{i}(x) \neq 0\right\} ;
$$

it is a finite or void subset of $J$. It is clear that any $F$-seminorm in $\mathcal{F}_{1}$ has the following property:

$$
\|x+y\|=\|x\|+\|y\| \quad \text { for all } x, y \in X \text { with } \operatorname{supp}(x) \cap \operatorname{supp}(y)=\emptyset .
$$

Proposition 1 ([3]). Let $X$ be a real or complex vector space provided with the topology $\tau_{\max }$. Then $X$ is a complete (Hausdorff) topological vector space.

Proof. Let $\left(x_{\alpha}\right)_{\alpha \in \mathfrak{a}}$ be a Cauchy net in $X$; we have to show that it is convergent to some element $x_{0}$. We can assume that the topology of 
$X$ is given by the above defined family $\mathcal{F}_{1}$. Clearly for every continuous linear functional $f$ on $X$ the net $\left(f\left(x_{\alpha}\right)\right)_{\alpha \in \mathfrak{a}}$ is also Cauchy. Since all linear functionals on $X$ are continuous the (finite) limits $a_{i}=\lim _{\alpha} g_{i}\left(x_{\alpha}\right)$ exist for all $i$ in $J$.

We claim that only finitely many numbers $a_{i}$ can be different from zero. If not, we have $a_{i_{n}} \neq 0$ for a sequence $\left(i_{n}\right)_{n=1}^{\infty}$ of (different) indices in $J$. Setting

$$
|x|_{a}=\sum_{n=1}^{\infty} 2 n\left|g_{i_{n}}(x)\right| /\left|a_{i_{n}}\right|
$$

we obtain a well defined (continuous) seminorm on $X$. Since for every (continuous) $F$-seminorm $|\cdot|$ the net $\left(\left|x_{\alpha}\right|\right)$ is also Cauchy, the (finite) limit $M=$ $\lim _{\alpha}\left|x_{\alpha}\right|_{a}$ exists. For a fixed natural $m$ there is an index $\alpha_{0} \in \mathfrak{a}$ such that $\left|g_{i_{m}}\left(x_{\alpha}\right)\right|>\left|a_{i_{m}}\right| / 2$ for all $\alpha \succeq \alpha_{0}$. Thus $\left|x_{\alpha}\right|_{a} \geq 2 m\left|g_{i_{m}}\left(x_{\alpha}\right)\right| /\left|a_{i_{m}}\right|>m$ for all $\alpha \succeq \alpha_{0}$. This implies $M \geq m$, and since $m$ was arbitrarily chosen, this gives a contradiction proving our claim.

Thus $x_{0}=\sum_{i \in J} a_{i} h_{i}$ is a well defined element of $X$. Setting $y_{\alpha}=x_{\alpha}-x_{0}$ we obtain a Cauchy net in $X$ with $\lim _{\alpha} g_{i}\left(y_{\alpha}\right)=0$ for all $i$ in $J$. Our conclusion will follow if we show that $\lim _{\alpha} y_{\alpha}=0$, because then $\lim _{\alpha} x_{\alpha}=$ $x_{0}$.

Suppose that $\left(y_{\alpha}\right)$ does not tend to zero. By the assumption there is an $F$-seminorm $|\cdot|_{0}$ in $\mathcal{F}_{1}$ with $M_{0}=\lim _{\alpha}\left|y_{\alpha}\right|_{0}>0$. We can now find an index $\alpha_{1} \in \mathfrak{a}$ such that

$$
\left|y_{\alpha}-y_{\alpha_{1}}\right|_{0}<M_{0} / 2 \quad \text { for all } \alpha \succeq \alpha_{1} .
$$

Put $J_{0}=\operatorname{supp}\left(y_{\alpha_{1}}\right)$ and define on $X$ a (continuous) projection

$$
P x=\sum_{i \in J_{0}} g_{i}(x) h_{i} .
$$

Clearly $\operatorname{supp}(P x) \cap \operatorname{supp}((I-P) x)=\emptyset$ and $\operatorname{supp}((I-P) x) \cap J_{0}=\emptyset$ for all $x \in X$, where $I$ is the identity operator on $X$. Applying to $|\cdot|_{0}$ the formula (5) we obtain

$$
\begin{aligned}
\left|y_{\alpha}-y_{\alpha_{1}}\right|_{0} & =\left|P y_{\alpha}-y_{\alpha_{1}}+(I-P) y_{\alpha}\right|_{0} \\
& =\left|P y_{\alpha}-y_{\alpha_{1}}\right|_{0}+\left|(I-P) y_{\alpha}\right|_{0} \geq\left|(I-P) y_{\alpha}\right|_{0},
\end{aligned}
$$

which, by (7), implies

$$
\left|(I-P) y_{\alpha}\right|_{0}<M_{0} / 2 \quad \text { for all } \alpha \succeq \alpha_{1} .
$$

Since $\lim _{\alpha} g_{i}\left(y_{\alpha}\right)=0$ for all $i$ and the set $J_{0}$ is finite, we see by the definition of the class $\mathcal{F}_{1}$ that $\lim \left|P y_{\alpha}\right|_{0}=0$. Thus (5) and (8) imply

$M_{0}=\lim _{\alpha}\left|y_{\alpha}\right|_{0}=\lim _{\alpha}\left|P y_{\alpha}\right|_{0}+\lim _{\alpha}\left|(I-P) y_{\alpha}\right|_{0}=\lim \left|(I-P) y_{\alpha}\right|_{0} \leq M_{0} / 2$.

This contradiction completes the proof. 
The following result was obtained by means of inductive limits; here we present an elementary proof.

Proposition 2 ([3], [11]). Let $X$ be a real or complex vector space. Then the topologies $\tau_{\max }^{\mathrm{LC}}$ and $\tau_{\max }$ coincide on $X$ whenever it is countabledimensional.

Pr o of. By assumption $X$ has a countable Hamel basis $\left(h_{i}\right)_{i=1}^{\infty}$. Let $|\cdot|_{0} \in$ $\mathcal{F}_{1}$. We shall be done if we show that $|\cdot|_{0}$ is continuous with respect to some (homogeneous) seminorm $\|\cdot\|$ on $X$. Thus we have to construct a seminorm $\|\cdot\|$ with the property that for each positive $\varepsilon$ there is a positive $\delta$ such that $\|x\|<\delta$ implies $|x|_{0}<\varepsilon$ for all $x$ in $X$. Let $\left(f_{i}\right)$ be the sequence of functions of class $\mathcal{S}$ giving $|\cdot|_{0}$ by means of $(2)$. Since $\lim _{t \rightarrow 0} f_{i}(t)=0$ for $i=1,2, \ldots$, there is a sequence $\left(a_{i}\right)$ of positive numbers such that

$$
\sum_{i=1}^{\infty} f_{i}\left(a_{i}\right) \leq 1
$$

Take a positive $\varepsilon$ and choose a natural $n_{0}$ so that

$$
\sum_{i=n_{0}+1}^{\infty} f_{i}\left(a_{i}\right)<\varepsilon / 2 .
$$

Since the $f_{i}$ are non-decreasing and tend to zero at 0 there is a positive $\delta \leq 1$ such that

$$
\sum_{i=1}^{n_{0}} f_{i}\left(\delta a_{i}\right)<\varepsilon / 2
$$

Define

$$
\|x\|=\sum_{i=1}^{\infty}\left|g_{i}(x)\right| / a_{i} .
$$

It is a (continuous) seminorm on $X$. Let $\|x\|<\delta$. By (12) we have $\left|g_{i}(x)\right|<$ $\delta a_{i} \leq a_{i}$ for all $i$ and so by (10) and (11) we obtain

$$
|x|_{0}=\sum_{i=1}^{\infty} f_{i}\left(\left|g_{i}(x)\right|\right) \leq \sum_{i=1}^{n_{0}} f_{i}\left(\delta a_{i}\right)+\sum_{i=n_{0}+1}^{\infty} f_{i}\left(a_{i}\right)<\varepsilon .
$$

The conclusion follows.

As a corollary we have a result of [5] obtained here in a much simpler way.

Corollary 3. Let $X$ be as above. Then all the topologies $\tau_{\max }^{p}$ and $\tau_{\max }^{q+}$, $0<p \leq 1,0 \leq q<1$, coincide with $\tau_{\max }^{\mathrm{LC}}$.

Proof. This follows immediately from the previous theorem and the relations $\tau_{\max }^{\mathrm{LC}} \leq \tau_{\max }^{p} \leq \tau_{\max }$ and $\tau_{\max }^{\mathrm{LC}} \leq \tau_{\max }^{q+} \leq \tau_{\max }$. 
As another corollary we obtain our main result:

THEOREM 4. Let $X$ be a real or complex vector space provided with the topology $\tau_{\max }$. Then each separable subspace of $X$ is locally convex.

P r o o f. Let $X_{0}$ be a separable subspace of $X$ with a dense subset $\left(x_{i}\right)_{i=1}^{\infty}$. Since all linear subspaces of $X$ are closed, we have $X_{0}=\operatorname{span}\left\{x_{i}\right\}$, so that $X_{0}$ is at most countable-dimensional. To obtain the conclusion it is sufficient to show that the relative topology of $X_{0}$ is again the topology $\tau_{\max }$, or that every $F$-seminorm of class $\mathcal{F}_{1}$ on $X_{0}$ extends to one on $X$. Without loss of generality we can assume that $X_{0}$ is countable-dimensional and take in it a Hamel basis $\left(h_{i}\right)_{i=1}^{\infty}$. Take any $F$-seminorm of class $\mathcal{F}_{1}$ on $X_{0}$ :

$$
|x|_{0}=\left|\sum_{i=1}^{\infty} g_{i}(x) h_{i}\right|_{0}=\sum_{i=1}^{\infty} f_{i}\left(\left|g_{i}(x)\right|\right) .
$$

Since $\left(h_{i}\right)$ extends to a Hamel basis on $X$, all $g_{i}$ can be viewed as functionals on $X$, and the same formula gives an $F$-seminorm of class $\mathcal{F}_{1}$ on $X$; we have thus obtained the desired extension. The conclusion follows.

It is known that for an uncountable-dimensional vector space $X$ the topologies $\tau_{\max }^{\mathrm{LC}}$ and $\tau_{\max }$ are different (see [11], p. 213). As was shown in [5], also all the topologies $\tau_{\max }^{p}$ and $\tau_{\max }^{q+}$ are mutually different in this case. We now show that they are also different from $\tau_{\max }$.

Proposition 5. Let $X$ be an uncountable-dimensional real or complex vector space. Then the topology $\tau_{\max }$ on $X$ is strictly stronger than each of the topologies $\tau_{\max }^{p}, \tau_{\max }^{q+}, 0<p \leq 1,0 \leq q<1$.

Proof. Since $\tau_{\max }^{0+}$ is the strongest of the topologies $\tau_{\max }^{p}, \tau_{\max }^{q+}$ it is sufficient to show that $\tau_{\max }$ is strictly stronger than $\tau_{\max }^{0+}$. To this end we shall construct an $F$-seminorm $\|\cdot\|_{0}$ which is discontinuous in the topology $\tau_{\max }^{0+}$, i.e. it is continuous with respect to no $p$-homogeneous seminorm on $X$ (any finite number $\|\cdot\|_{1}, \ldots,\|\cdot\|_{n}$ of $p_{i}$-homogeneous seminorms are each continuous with respect to the $p$-homogeneous seminorm $\|x\|=\max \left\{\|x\|_{1}^{p / p_{1}}, \ldots,\|x\|_{n}^{p / p_{n}}\right\}$, where $\left.p=\min \left\{p_{1}, \ldots, p_{n}\right\}\right)$.

Define

$$
q(t)= \begin{cases}1 /|\log t|^{1 / 2} & \text { if } 0<t \leq e^{-1} \\ 1 & \text { if } t \geq e^{-1},\end{cases}
$$

and put $f(t)=t^{q(t)}$ for $t>0$ and $f(0)=0$. It is easy to verify that $f$ is in the class $\mathcal{S}$ and

$$
\lim _{t \rightarrow 0} f(t) / t^{p}=\infty \quad \text { for each } p>0
$$


Fix a Hamel basis $\left(h_{i}\right)_{i \in J}$ for $X$ and put

$$
\|x\|_{0}=\sum_{i \in J} f\left(\left|g_{i}(x)\right|\right) ;
$$

it is an $F$-seminorm on $X$. Assume that $\|\cdot\|_{0}$ is continuous with respect to some $p$-homogeneous seminorm $|\cdot|$. We have

$$
|x|=\left|\sum_{i \in J} g_{i}(x) h_{i}\right| \leq \sum_{i \in J}\left|g_{i}(x)\right|^{p} r_{i}=\|x\|_{r},
$$

where $r_{i}=\left|h_{i}\right|$ and the $p$-homogeneous seminorm $\|\cdot\|_{r}$ is defined by the righthand equality. Clearly $\|\cdot\|$ must also be continuous with respect to $\|\cdot\|_{r}$. Since $J$ is uncountable, there is a natural $k$ such that the set $J_{k}=\left\{i \in J: r_{i} \leq k\right\}$ is infinite. By the assumption there is a positive $\delta$ such that $\|x\|_{0}<1$ whenever $\|x\|<\delta$. Choose a natural $n$ so that

$$
n \delta>2 \text {. }
$$

By (13) there is a positive $t_{0}$ such that

$$
f\left(t_{0}\right)>n k t_{0}^{p}
$$

and

$$
k t_{0}^{p}<\delta / 4
$$

Let $s$ be the largest integer for which $s k t_{0}^{p}<\delta$. By (16) we have

$$
\delta / 2<s k t_{0}^{p}<\delta .
$$

Choose arbitrarily $i_{1}, \ldots, i_{s}$ in $J_{k}$ and put $x_{0}=\sum_{j=1}^{s} t_{0} h_{i_{j}}$. By (17) we have

$$
\left\|x_{0}\right\|_{r}=\sum_{i=1}^{s} t_{0}^{p} r_{i_{j}} \leq s t_{0}^{p} k<\delta
$$

so that we should have $\left\|x_{0}\right\|_{0}<1$. But by (14), (15) and (17) we obtain

$$
\left\|x_{0}\right\|_{0}=s f\left(t_{0}\right)>s n k t_{0}^{p}>n \delta / 2>1,
$$

which gives a contradiction. The conclusion follows.

COROLlaRY 6. The topology $\tau_{\max }$ is not locally pseudoconvex on a vector space $X$ if and only if the dimension of $X$ is uncountable.

\section{References}

[1] S. Banach, Théorie des Opérations Linéaires, Warszawa, 1932.

[2] N. Dunford and J. T. Schwartz, Linear Operators I, Interscience, New York, 1958.

[3] H. Jarchow, Locally Convex Spaces, Teubner, Stuttgart, 1981.

[4] A. Grothendieck, Topological Vector Spaces, Gordon and Breach, New York, 1973. 
[5] A. Kokk and W. Żelazko, On vector spaces and algebras with maximal locally pseudoconvex topologies, Studia Math. 112 (1995), 195-201.

[6] G. Köthe, Topological Vector Spaces I, Springer, Berlin, 1969.

[7] —, Topological Vector Spaces II, Springer, Berlin, 1979.

[8] S. Rolewicz, Metric Linear Spaces, PWN, Warszawa, 1972.

[9] H. H. Schaefer, Topological Vector Spaces, Springer, New York, 1971.

[10] L. Waelbroeck, Topological Vector Spaces and Algebras, Lecture Notes in Math. 230, Springer, 1971.

[11] A. Wilansky, Modern Methods in Topological Vector Spaces, McGraw-Hill, New York, 1978.

Institute of Mathematics

Polish Academy of Sciences

Śniadeckich 8

P.O. Box 137

00-950 Warszawa, Poland

E-mail: zelazko@impan.impan.gov.pl 